\title{
Low-loss polyacrylate dielectric elastomer with outstanding actuation performance by optimizing crosslinking network
}

\section{Li-Juan Yin}

Tsinghua University

Yu Zhao

Zhenzhou University

Jing Zhu

Tsinghua University

Minhao Yang

North China Electric Power University

Jia-Yao Pei

Tsinghua University

Shao-Long Zhong

Tsinghua University

Zhi-Min Dang ( $\nabla$ dangzm@tsinghua.edu.cn )

Tsinghua University

\section{Article}

Keywords:

Posted Date: November 4th, 2020

DOI: https://doi.org/10.21203/rs.3.rs-96989/v1

License: (c) (i) This work is licensed under a Creative Commons Attribution 4.0 International License. Read Full License

Version of Record: A version of this preprint was published at Nature Communications on July 26th, 2021. See the published version at https://doi.org/10.1038/s41467-021-24851-w. 


\section{Abstract}

This work reports the low-loss polyacrylate dielectric elastomer with outstanding actuation performance by employing a molecular engineering route. It is found that the difunctional macromolecular crosslinking agent we choose can not only effectively tune the dimensions of elastomer meshes and avoid uneven distribution of crosslinking points in elastomer matrix, accordingly eliminating the stress concentration and delaying the failure of elastomer, but also as "soft segments" and alleviate the strong dipole-dipole interaction between adjacent side groups of polyacrylate, which makes the dielectric elastomer with low elastic modulus and low mechanical loss. Simultaneously, a certain amount of uncrosslinked chains will remain in the free volume space of elastomer network, which would increase the dielectric constant of assynthesized elastomers owing to their strong orientation ability. Therefore, the improvements in mechanical and dielectric properties of elastomer will contribute to the amelioration of actuation performance. We also design a home-made non-magnetic motor apparatus to verify the optimized actuation performance. This finding represents the first molecular engineering approach to improve the intrinsic actuation performance of dielectric elastomers, which are key materials of choice for flexible dielectric elastomer actuators.

\section{Introduction}

The eternal topics for aerospace and soft robotics are light weight and flexibility, which are almost inaccessible for conventional electromagnetic motors and pneumatic actuators ${ }^{1,2}$. Dielectric elastomer actuators (DEAs) with large electrically actuated strain $^{3-5}$ could be used to realize the light-weight and flexible non-magnetic motors ${ }^{6-8}$. Dielectric elastomers in soft actuation field usually suffer from low dielectric constant ${ }^{9}$, high stiffness ${ }^{10}$, and low actuation sensitivity ${ }^{11,12}$, which severely affect the electromechanical energy conversion performance of DEAs ${ }^{13}$. Here we report a new polyacrylate dielectric elastomer with optimized polymer network by rationally employing difunctional macromolecular crosslinking agent via a simple UV curing synthesis process. The synthesized acrylic elastomers exhibit relatively high dielectric constant (5.75@1kHz), ultra-low dielectric loss (tan $\mathrm{d}<0.002$ @ 1kHz) and mechanical loss, and desirable Young's modulus ( 0.088 MPa), which are superior to commercial $3 \mathrm{M}$ $\mathrm{VHB}^{\mathrm{TM}}$ 4910. Accordingly, huge actuation strain (118\% @70 $\left.\mathrm{MV} \mathrm{m}^{-1}\right)$ and rapid response are successfully achieved in this elaborate polyacrylate elastomer. Besides, a home-made motor made of synthesized polyacrylate dielectric elastomer could be driven under a low electric field of $32 \mathrm{MV} \mathrm{m}^{-1}$ and high frequency. Our work reveals that a molecular engineering route reasonably realizes the low-loss polyacrylate dielectric elastomer with outstanding actuation performance. However, this strategy has never been used to adjust the properties of dielectric elastomers, which hold great promise for ingenious and flexible DEAs.

As the milestone equipment of the Second Industrial revolution, electromotors have greatly motivated the advance of technology and society. Up to now, the intrinsic features of electromotors like low specific power, bulky frame, rigid structure and complicated control system, cannot meet the requirement of light 
weight, high flexibility and easy to control for soft micromotors, which have a wide application in flexible electronics ${ }^{14,15}$, soft robotics ${ }^{16,17}$, biomedical implantation ${ }^{18}$ and aerospace ${ }^{19,20}$. Recently, soft actuators made of diverse electro-active polymers (EAPs) with electrically actuated deformation have received considerable attention in aforementioned fields ${ }^{5,9,12,21-23}$. Different from the actuators with ferroelectric polymers $^{21,23}$, dielectric elastomer actuator (DEA), an electrically stimulus-responsive actuator, are of great interest for the large strain and superior flexibility 4,5,22,24. A large variety of commercial elastomers, including silicone rubbers ${ }^{9,25-27}$, polyurethanes ${ }^{28}$, and acrylic elastomers (VHB ${ }^{\mathrm{TM}} 4910$ from $\left.3 \mathrm{M}\right)^{5,8}$, have been widely used to assemble DEA. Among them, acrylic-based elastomers stand out for the fabrication of non-magnetic motors because of their high dielectric constant ( 4.4@1kHz), large area strain (>380\%) and high energy density $\left(3.4 \mathrm{MJ} \mathrm{m}^{-3}\right)^{29}$. However, commercial $\mathrm{VHB}^{\mathrm{TM}}$ acrylic elastomers usually require high driven electric field $(E)\left(>80 \mathrm{MV} \mathrm{m}^{-1}\right)^{6-8}$, and exhibit slow response speed and serious viscoelastic creep due to their inherently high stiffness and viscoelastic loss ${ }^{30}$.

Numerous efforts have been made to reduce the driven $E$ and increase the response speed of acrylicbased elastomer ${ }^{31,32}$. According to the Maxwell stress, the actuation strain $\left(S_{z}\right)^{5}$ in the thickness direction can be defined by, where $e_{0}, e_{n} Y$ and $E$ are the dielectric constant of vacuum, dielectric constant of elastomer, Young's modulus of elastomer and applied electric field, respectively. Thus, the driven $E$ under a fixed actuation strain can be reduced by enhancing the actuation sensitivity $(b$,$) , which is usually$ achieved by either increasing the $e_{r}$ or decreasing the $Y$ of the elastomer ${ }^{9,11,12,31}$. A common strategy to increase the $e_{r}$ of elastomer used is to incorporate fillers with high dielectric constant into the elastomer matrix ${ }^{11,33-35}$. However, high filler concentration is a requisite for achieving a considerable increase of $e_{n}$ which, in turn, makes the elastomer stiff ${ }^{33}$ and usually causes premature electrical breakdown due to serious agglomeration of fillers and inferior compatibility between fillers and polymer matrix ${ }^{35}$. Adding plasticizers with short molecular chains into the elastomer matrix is a typical way to decrease the $Y$ of the elastomers, which can promote the rotation of elastomer chains and reduce the glass transition temperature $\left(T_{g}\right)$ of the elastomer, thereby broadening the working temperature of the elastomer $29,32,36$. Nevertheless, the poor compatibility between the guest and host may lead to the leakage of plasticizers under a harsh or multi-cycled environment. As for the response speed, it can be improved by decreasing the mechanical loss of elastomer stemming from an amount of inter-molecular interaction ${ }^{7}$. Reducing the density of large steric hindrance groups and polar groups can significantly suppress the mechanical loss of elastomers. However, the suppression of the mechanical loss is usually accompanied by the decrease of $e_{r}^{26}$.

In this contribution, we report a strategy to prepare low-loss polyacrylate dielectric elastomer with outstanding actuation performance by optimizing crosslinking network. Herein, we choose $n$-butyl acrylate (BA) as the monomers, theoretically filtered by the Quantitative Structure-Property Relationship (QSPR) approach ${ }^{37}$, and optimize the network of photo-polymerized acrylic-based elastomer employing a large molecular weight urethane acrylate compound (CN9021NS) as crosslinker with flexible polyether 
diol and aliphatic diisocyanate segments ${ }^{32}$. Macro-molecular crosslinker (CN9021NS) with two vinyl groups at chain ends, not only provides crosslinking points for polymer network, but also acts as part of network to form a hybrid network. The flexible long chain structure of macromolecular crosslinkers can effectively tune the dimensions of elastomer meshes and avoid uneven distribution of crosslinking points in elastomer matrix, accordingly eliminating the stress concentration and delaying the failure of elastomer. Additionally, the flexible polyether diol segment of macromolecular crosslinkers can also act as "soft segments" and alleviate the strong dipole-dipole interaction between adjacent side groups of poly (BA), which is helpful to achieving low elastic modulus and mechanical loss. More importantly, long chain structure of macromolecular crosslinkers will reduce the reactive probability of the crosslinking groups due to the steric hindrance effect. Thereby, a certain amount of uncrosslinked chains will remain in the free volume space of elastomer network, which would increase the dielectric constant of assynthesized elastomers owing to their strong orientation ability. Therefore, the improvements in mechanical and dielectric properties of elastomer will contribute to the amelioration of actuation performance. We also design a home-made motor apparatus to verify the optimized actuation performance.

\section{Results And Discussion}

The crosslinked network of elastomer has a significant influence on the intrinsic mechanical and electrical properties of dielectric elastomer, accordingly affecting its actuation behavior achieved by the conversion of electrical to mechanical energy. The polymer network is usually established by the chemical bonding between the polymer main chains and crosslinking agent with multiple reactive groups or the physical entanglement of macromolecular chains. Therefore, the uneven dispersion of micromolecular crosslinkers causes the uneven distribution of cross-linking points which plays a vital role in determining the uniformity of polymer network (Fig. 1ai and 1aii). In this contribution, we innovatively develop a strategy for the optimization of crosslinked polymer network through modulating the dimension of crosslinking agents which can weaken the dependence of uniformity of network on crosslinker dispersion (Fig. 1aiii and 1aiv). In addition to good compatibility or solubility (dispersion) of crosslinkers in polymer or precursor matrix, appropriate average molecular weight (dimension) also facilitates the formation of a uniform crosslinked network. Here, the monomer used for the polymerization of acrylic elastomer and corresponding crosslinking agent were theoretically selected to acquire a uniform crosslinked polymer network (see Extended Data, Part: "Selection of number average molecular weight"). After that, the monomer of $n$-butyl acrylate (BA) was adopted for the fabrication of acrylic elastomer due to the good flexibility, appropriate intermolecular interaction, and broad working temperature range of $n$-butyl acrylate homopolymer (Extended Data Fig. 1). Additionally, the oligomer with polyether as repeat units was recommended for serving as the crosslinking agent due to its good flexibility and solubility in BA monomer matrix (see Extended Data, Part: "Network design", Extended Data Fig. 2). 
The average molecule weight of crosslinker should also be in the range of $10^{4} \mathrm{~g} \mathrm{~mol}^{-1}$ to $10^{5} \mathrm{~g} \mathrm{~mol}^{-1}$, which matches the range of average molecular weight ( ) between crosslinking points (see Extended Data, Part: "Selection of number average molecular weight"). Considering the analysis discussed above, CN9021NS, a difunctional urethane acrylate compound composed of a flexible polyether diol segment and an aliphatic diisocyanate segment, was chosen as macromolecular crosslinker for the construction of $n$-butyl acrylicbased elastomer network (BAC). For comparison, $n$-butyl acrylate homopolymer (BAP), crosslinked by the equimolar (taking BAC2 as reference) polyethylene glycol (400) diacrylate (a small molecular crosslinker), was also prepared through a similar polymerization process. The terminal vinyl groups (-CH₫CH2) of CN9021NS were chemically bonded into the adjacent polymer main chains during the photo-polymerization process and a structure-controlled and uniform hybrid network was subsequently obtained (Fig. 1aiv). Accordingly, as shown in Fig. 1b and 1c, the BAC2 specimen exhibits great stretchability ( 2300\%), much higher than that of BAP. Meanwhile, the BAC2 sample owns a low elastic modulus (0.088MPa), which is attributed to the existence of long polyether diol segment in CN9021NS. The long polyether diol segment serves as a "soft segment" in the crosslinked network and provides the network with excellent flexibility. It is a huge challenge to simultaneously improve the softness and toughness of elastomer and such a paradox is solved in this contribution through the optimization of crosslinked network. The BAC2 sample not only exhibits a low Young's modulus and a high elongation but also displays a high toughness (6.77 $\mathrm{MJ} \mathrm{m}^{-3}$ ) and a high strength (1.34 MPa) (Fig. 1d and 1e, Extended Data Fig. 4). The excellent mechanical property displayed here is seldom observed in previously reported works.

A crosslinked polymer network is usually composed of the crosslinked part and an appropriate amount of dissociative chains that are not involved in the construction of network (Fig. 2a). The proportion of dissociative chains in the network crosslinked by the macromolecular agent is probably increased due to the "cage effect" and steric hindrance of macromolecular chains, which significantly weakens the reactive probability of terminal groups of macromolecular crosslinking agents (CN9021NS). Thus, the crosslinking efficiency and density will be subsequently suppressed. Such a hypothesis is verified by the swell experiment and the result indicates that BAC2 sample owns the highest fraction of dissociative component (18 \%) when compared with $\mathrm{VHB}^{\mathrm{TM}} 4910$ and other BAC samples (Fig. 2b). The analysis of the element and molecular weight distribution of CN9021NS and free chains extracted from BAC2 sample after swelling experiment indicates that the dissociative component is mainly composed of unreacted CN9021NS and uncrosslinked poly(n-butyl acrylate) chains (Fig. 2c and 2d).

We observe that the effect of these free chains inside the crosslinked network on the actuation performance of elastomer should not be neglected. The dielectric and mechanical 
properties of elastomer are of great importance for the actuation performance of dielectric elastomer. Dielectric analysis of VHB ${ }^{\mathrm{TM}} 4910$ and BAC samples was carried out and the result shows that BAC2 sample exhibits the highest dielectric constant value when compared with those of VHB ${ }^{\mathrm{TM}} 4910$ and other BAC samples (Fig. 2e and Extended Data Fig. 5b). This is out of our expectation since the long flexible polyether segment in CN9021NS may contribute to the decrease of dielectric constant for BAC2 because of its lower dipole density compared with that of poly( $n$-butyl acrylate $)^{32}$. As for the loss part, BAC samples exhibit significant low dielectric loss than that of $\mathrm{VHB}^{\mathrm{TM}} 4910$, and no obvious change for the loss of BAC series samples is observed (Fig. 2e and Extended Data Fig. 5b). In order to figure out the contribution of the free chains inside the crosslinked network to the dielectric properties of elastomer, the comparison of the dielectric properties of $\mathrm{VHB}^{\mathrm{TM}} 4910$ and BAC2 samples before and after swelling experiment was performed. Compared with the change of $\mathrm{VHB}^{\mathrm{TM}} 4910$, the dielectric constant of BAC2 sample after swollen displays an obvious decrease from 5.75 for the original one to 5.4 while the loss almost remains unchanged (Fig. 2e).

Additionally, the comparison of storage modulus and mechanical loss before and after swollen indicates the existence of these free chains does not raise the negative effect on the mechanical properties of elastomer (Fig. $2 \mathrm{f}$ and $2 \mathrm{~g}$ ). Different from those conventional strategies implemented by incorporating high dielectric constant fillers into elastomer matrix or grafting polar groups into elastomer main chains, the approach employed in this contribution apparently eliminates the occurrence of the inevitable deterioration of dielectric loss and mechanical properties of elastomer. More importantly, these free chains inside the elastomer matrix are helpful to improve the dielectric and mechanical properties of elastomer, accordingly ameliorating actuation performance. The contribution of these free chains to the dielectric and mechanical properties of elastomer could be explained by the following two aspects. Firstly, the free chains have more orientation freedom than that of constrained segments in crosslinked network. These free chains not only afford the elastomer strong polarization intensity under external electric field, but also increase the density of dipoles in elastomer matrix (Fig. 2aiii and 2aiv). Therefore, dielectric properties of elastomers with dissociative chains is significantly improved (Fig. 2e). Secondly, the mechanical properties of elastomer mainly depend on the crosslinked network. Actually, these free chains have not been involved in the construction of the polymer network. Thus, no significant change of mechanical properties is observed (Fig. $2 \mathrm{f}$ and $2 \mathrm{~g}$ ). More importantly, for the mechanical loss obtained from the cyclic stress-strain curve, the loss of BAC2 sample is much lower than that of $\mathrm{VHB}^{\mathrm{TM}} 4910$ (Fig. 2h), which may facilitate the improvements in electro-mechanical conversion efficiency and driving response speed of elastomer. The flexible long aliphatic polyether backbone inside CN9021NS can act as the "spacer" among adjacent polyacrylate chains and the introduced "spacer" will weaken 
dipole-dipole interaction, according reducing mechanical energy loss during deformation process. The suppressed loss obtained here is extremely important for the reduction of heat production during the cyclic deformation process.

When dielectric elastomers are used for actuators, actuation sensitivity $(b)$ is one of the most important parameters that is usually used to evaluate the actuation performance and is defined as the ratio of dielectric constant and Young's modulus. As discussed previously, the dielectric constant of BAC2 sample is the highest value among those commercial and lab-made samples, whereas its Young's modulus is obviously lower than that of $\mathrm{VHB}^{\mathrm{TM}}$ 4910. Accordingly, owing to the high and low $Y$, the actuation sensitivity of BAC2 elastomer film can reach up to 65 , which is 2.25 times larger than that (20) of $\mathrm{VHB}^{\mathrm{TM}} 4910$ (Fig. 3a). In order to clearly evaluate the actuation performance of elastomers, a homemade experimental apparatus was specially designed (Extended Data Fig. 6). Due to the improved actuation sensitivity, BAC2 elastomer exhibits the largest area strain of $18.5 \%$ at $15 \mathrm{MV} \mathrm{m}^{-1}$ without pre-strain. Nevertheless, the area strain obtained from $\mathrm{VHB}^{\mathrm{TM}} 4910$ film at the same electric field is only $4.5 \%$ (Fig. 3b). When the films were stretched with $400 \%$ biaxial pre-strain, the area strain achieved in BAC2 elastomer film reaches up to $118 \%$ at $70 \mathrm{MV} \mathrm{m}^{-1}$, which is almost 3.5 times larger than that of commercial one at the same electric field (Fig. 3c). It is well known that the application of pre-strain on the elastomer film can not only suppress electromechanical instability, but also enhance electrical breakdown strength, consequently leading to the increase of maximum actuation strain at a higher electric field. Additionally, pre-strain can also be used to alleviate the viscoelasticity of poly-acrylate elastomers, which is regarded as the main issue for obtaining fast response speed and stabilizing strain under the applied electric field. As shown in Fig. 3d, the VHB ${ }^{\mathrm{TM}} 4910$ elastomer needs almost 5 min to reach $90 \%$ of its final strain, whereas BAC2 elastomer only needs $35.2 \mathrm{~s}$ to reach the same relative strain value (90\%). In addition to the fast response speed, the area strain of BAC2 elastomer is almost invariable after the application of applied electric field. On the contrary, the commercial one displays a severe creep behavior (Fig. 3d and Extended Data Fig. 7). The large area strain and rapid electromechanical response make BAC2 elastomer film as a promising candidate in the actuation fields with the requirements of low electric field operation and potential broad frequency output.

Considering the superior dielectric, mechanical, and actuation performance of BAC2 film, the motor made of BAC2 film may rotate at a lower electric field and in a broader frequency range compared with the one using $\mathrm{VHB}^{\mathrm{TM}} 4910$ film. Fig. 4a displays a complete rotation process for the BAC2 based motor. As illustrated in Fig. 4b, the rotation speed exhibits a monotonous increase with driving frequency under a fixed electric field. Besides, the maximum rotation speed increases obviously when the electric field is enhanced. The 
minimum driving electric field is a vitally important parameter in evaluating the actuation performance of elastomer film. The minimum driving electric field is significantly reduced from $48 \mathrm{MV} \mathrm{m}^{-1}$ for $\mathrm{VHB}^{\mathrm{TM}} 4910$ based motor to $32 \mathrm{MV} \mathrm{m}^{-1}$ for BAC2 based one, which is attributed to the improved actuation sensitivity. Additionally, owing to the fast response, BAC2 based motor could be triggered to rotate at higher frequency than $\mathrm{VHB}^{\mathrm{TM}} 4910$ based motor (Fig. 4b). At the same driving electric field (48 $\mathrm{MV} \mathrm{m}^{-1}$ ), the maximum rotation rate of BAC2 based motor is $0.72 \mathrm{r} \mathrm{s}^{-1}$, which is 16 times larger than that of VHB ${ }^{\mathrm{TM}} 4910$ based motor. Furthermore, the rotation rate of the motor could be further increased to 2.86 $\mathrm{r} \mathrm{s}^{-1}$ when equipped with transmission gears (Fig. 4c, Supplementary Videos 1, 2 and 3). The output torque and power of motors mainly depend on the Maxwell pressure, strain, and mechanical loss of elastomers. The Maxwell pressure on the elastomer film scales linearly with dielectric constant and quadratically with the applied electric field while the strain is defined as the ratio of Maxwell pressure and Young's modulus.[12] The increase of the Maxwell pressure and strain usually indicates that more mechanical energy is converted from electric energy. Similarly, the less mechanical loss implies more power output. Due to the significant improvement of dielectric constant, mechanical loss and actuation performances as discussed above, the output torque and power of BAC2 based motor are distinctly improved (Fig. 4d). The output torque and power are 6 times and 18 times larger than those of $\mathrm{VHB}^{\mathrm{TM}} 4910$ based motor, respectively. The fabrication process and characterization of these soft motors are displayed in the Extended Data section in detail. Limited by the experimental equipment and dimension of motors, time constant for charging is at the magnitude of $10^{-2} \mathrm{~s}$. Thus, with the rise of driving frequency, the maximum voltage on film descends severely. Simulation from MATLAB/Simulink reveals that the output performance of $\mathrm{VHB}^{\mathrm{TM}} 4910$ based motor depends merely on elastomer itself. However, the output performance of BAC2 based motor is not only related to the elastomer but also restricted by the experiment platform (see Extended Data, Part: "Calculation of transient time constant and simulation of electrically driving frequency", and Extended Data Figs. 9 and 10). As a result, a great of key issues associated with high actuation voltage, slow response speed and severe viscoelastic drift have been resolved in this contribution. The approach developed here through the rational optimization of crosslinked elastomer network provides an effective strategy to tackle these challenges fundamentally.

In conclusion, a novel UV-curing polyacrylate dielectric elastomer with outstanding actuation performance was successfully obtained by optimizing polymer network. The monomer BA and macromolecular crosslinking agent CN9021NS were theoretically selected and a uniform polymer network was innovatively realized due to long chain structure of crosslinking agent used. Compared with $\mathrm{VHB}^{\mathrm{TM}} 4910$, the obtained polyacrylate 
elastomer here exhibits excellent dielectric (high dielectric constant), mechanical (low elastic modulus), and actuation performances (high actuation sensitivity). The simultaneous optimization of the dielectric and mechanical properties of elastomer film, a huge challenge for the conventional elastomers incorporated with high dielectric constant ceramic fillers or grafted with strong polar groups, is successfully achieved by the strategy developed in this work. The optimized actuation performance is also experimentally verified by a homemade apparatus. The performance obtained here could be mainly attributed to the flexible long chain structure of crosslinking agent and the existence of dissociative chains inside the network. The principle for the selection of elastomer component and strategy employed here provides a new insight for developing high-performance dielectric elastomers and actuators.

\section{Reference}

1. Mosadegh, B. et al. Pneumatic Networks for Soft Robotics that Actuate Rapidly. Funct. Mater. 24, 2163-2170, (2014).

2. Wehner, M. et al. An integrated design and fabrication strategy for entirely soft, autonomous robots. Nature 536, 451-455, (2016).

3. Zhao, X. \& Suo, Z. Theory of Dielectric Elastomers Capable of Giant Deformation of Actuation. Rev. Lett. 104, 178302, (2010).

4. Suo, Z. THEORY OF DIELECTRIC ELASTOMERS. Acta Mech. Solida Sin. 23, 549-578 (2010).

5. Pelrine, R., Kornbluh, R., Pei, Q. B. \& Joseph, J. High-speed electrically actuated elastomers with strain greater than 100\%. Science 287, 836-839, (2000).

6. Anderson, I. A. et al. A thin membrane artificial muscle rotary motor. Phys. A: Mater. Sci. Process. 98, 75-83, (2010).

7. Anderson, I. A. et al. A soft and dexterous motor. Appl. Phys. Lett. 98, 123704, (2011).

8. O'Brien, B. M., McKay, T. G., Gisby, T. A. \& Anderson, I. A. Rotating turkeys and self-commutating artificial muscle motors. Phys. Lett. 100, 074108, (2012).

9. Zhang, L. et al. Highly improved electro-actuation of dielectric elastomers by molecular grafting of azobenzenes to silicon rubber. Mater. Chem. C3, 4883-4889, (2015).

10. Ducrot, E. \& Creton, C. Characterizing Large Strain Elasticity of Brittle Elastomeric Networks by Embedding Them in a Soft Extensible Matrix. Funct. Mater. 26, 2482-2492, (2016).

11. Zhao, H., Wang, D.-R., Zha, J.-W., Zhao, J. \& Dang, Z.-M. Increased electroaction through a molecular flexibility tuning process in TiO2-polydimethylsilicone nanocomposites. Mater. Chem. A 1, 31403145, (2013).

12. Zhao, H., Zhang, L., Yang, M.-H., Dang, Z.-M. \& Bai, J. Temperature-dependent electro-mechanical actuation sensitivity in stiffness-tunable $\mathrm{BaTiO3/polydimethylsiloxane} \mathrm{dielectric} \mathrm{elastomer}$ nanocomposites. Phys. Lett. 106, (2015). 
13. Shian, S., Bertoldi, K. \& Clarke, D. R. Dielectric Elastomer Based "Grippers" for Soft Robotics. Mater. 27, 6814-6819, (2015).

14. Gao, W. et al. Fully integrated wearable sensor arrays for multiplexed in situ perspiration analysis. Nature 529, 509-514, (2016).

15. Rogers, J. A., Someya, T. \& Huang, Y. Materials and Mechanics for Stretchable Electronics. Science 327, 1603-1607, (2010).

16. Gu, G., Zou, J., Zhao, R., Zhao, X. \& Zhu, X. Soft wall-climbing robots. Robot. 3, eaat2874, (2018).

17. Li, T. et al. Agile and Resilient Insect-Scale Robot. Soft Robot. 6, 133-141, (2019).

18. Park, C. et al. An organosynthetic dynamic heart model with enhanced biomimicry guided by cardiac diffusion tensor imaging. Robot. 5, eaay9106, (2020).

19. Wang, W. \& Ahn, S.-H. Shape Memory Alloy-Based Soft Gripper with Variable Stiffness for Compliant and Effective Grasping. Soft Robot. 4, 379-389, (2017).

20. Ware, T. H., McConney, M. E., Wie, J. J., Tondiglia, V. P. \& White, T. J. Voxelated liquid crystal elastomers. Science 347, 982-984, (2015).

21. Huang, C. \& Zhang, Q. M. Enhanced dielectric and electromechanical responses in high dielectric constant all-polymer percolative composites. Funct. Mater. 14, 501-506, (2004).

22. Carpi, F., Bauer, S. \& De Rossi, D. Stretching Dielectric Elastomer Performance. Science 330, 17591761, (2010).

23. Qiao, B., Wang, X., Tan, S., Zhu, W. \& Zhang, Z. Synergistic Effects of Maxwell Stress and Electrostriction in Electromechanical Properties of Poly(vinylidene fluoride)-Based Ferroelectric Polymers. Macromolecules 52, 9000-9011, (2019).

24. Gu, G.-Y., Zhu, J., Zhu, L.-M. \& Zhu, X. A survey on dielectric elastomer actuators for soft robots. Bioinspiration Biomimetics 12, 011003, (2017).

25. Caspari, P., Dunki, S. J., Nuesch, F. A. \& Opris, D. M. Dielectric elastomer actuators with increased dielectric permittivity and low leakage current capable of suppressing electromechanical instability. Mater. Chem. C 6, 2043-2053, (2018).

26. Liu, X., Yu, L., Nie, Y. \& Skov, A. L. Silicone Elastomers with High-Permittivity lonic Liquids Loading. Eng. Mater. 21, 1900481, (2019).

27. Perju, E., Shova, S. \& Opris, D. M. Electrically Driven Artificial Muscles Using Novel Polysiloxane Elastomers Modified with Nitroaniline Push-Pull Moieties. ACS Appl. Mater. Interfaces 12, $23432-$ 23442, (2020).

28. Yin, G. et al. Dielectric Elastomer Generator with Improved Energy Density and Conversion Efficiency Based on Polyurethane Composites. ACS Appl. Mater. Interfaces 9, 5237-5243, (2017).

29. Brochu, P. \& Pei, Q. Advances in Dielectric Elastomers for Actuators and Artificial Muscles. Rapid Commun. 31, 10-36, (2010).

30. Maffli, L., Rosset, S., Ghilardi, M., Carpi, F. \& Shea, H. Ultrafast All-Polymer Electrically Tunable Silicone Lenses. Funct. Mater. 25, 1656-1665, (2015). 
31. Zhao, Y. et al. Remarkable electrically actuation performance in advanced acrylic-based dielectric elastomers without pre-strain at very low driving electric field. Polymer 137, 269-275, (2018).

32. Niu, X. et al. Synthesizing a new dielectric elastomer exhibiting large actuation strain and suppressed electromechanical instability without prestretching. J. Polym. Sci. Part B: Polym. Phys. 51, 197-206, (2013).

33. Ning, N. et al. Tailoring Dielectric and Actuated Properties of Elastomer Composites by Bioinspired Poly(dopamine) Encapsulated Graphene Oxide. ACS Appl. Mater. Interfaces 7, 10755-10762, (2015).

34. Yang, Y. et al. Enhanced energy conversion efficiency in the surface modified BaTiO3 nanoparticles/polyurethane nanocomposites for potential dielectric elastomer generators. Nano Energy 59, 363-371, (2019).

35. Zheng, M. S. et al. Improved dielectric, tensile and energy storage properties of surface rubberized BaTi03/polypropylene nanocomposites. Nano Energy 48, 144-151, (2018).

36. Black, A. L., Lenhardt, J. M. \& Craig, S. L. From molecular mechanochemistry to stress-responsive materials. Mater. Chem. 21, 1655-1663, (2011).

37. Bicerano, J. Prediction of Polymer Properties, 3rd Edition. (2002).

38. Shi, L. et al. Dielectric gels with ultra-high dielectric constant, low elastic modulus, and excellent transparency. NPG Asia Mater. 10, 821-826, (2018).

39. Zhao, Y. et al. Constructing advanced dielectric elastomer based on copolymer of acrylate and polyurethane with large actuation strain at low electric field. Polymer 149, 39-44, (2018).

40. Sheima, Y., Caspari, P. \& Opris, D. M. Artificial Muscles: Dielectric Elastomers Responsive to Low Voltages. Rapid Commun. 40, 1900205, (2019).

41. Wallin, T. J. et al. 3D printable tough silicone double networks. Commun. 11, 4000, (2020).

\section{Figures}




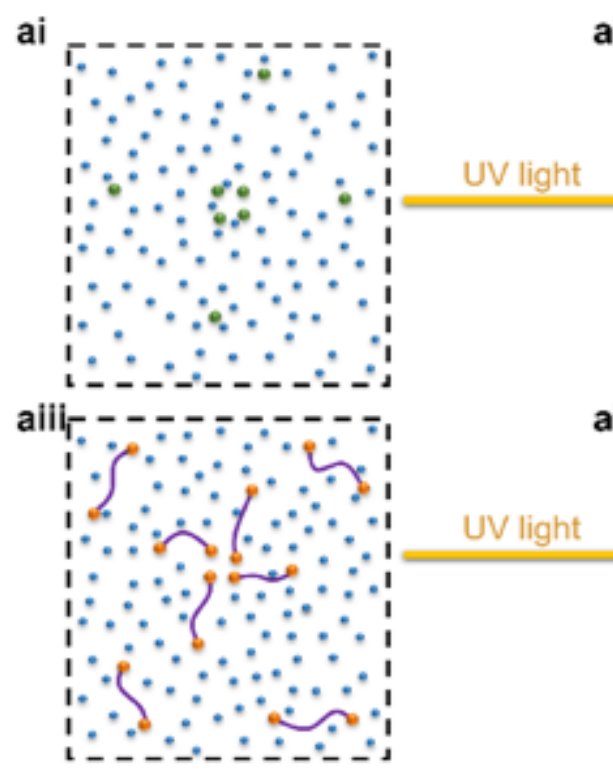

aii

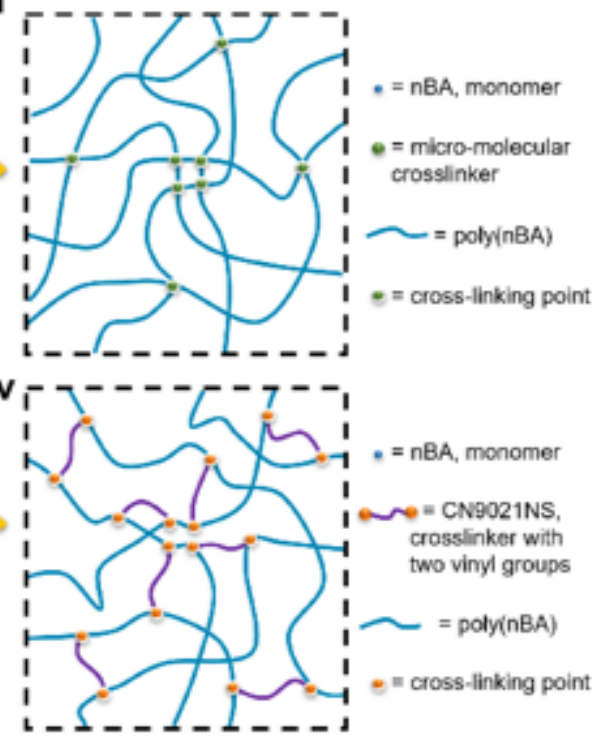

b

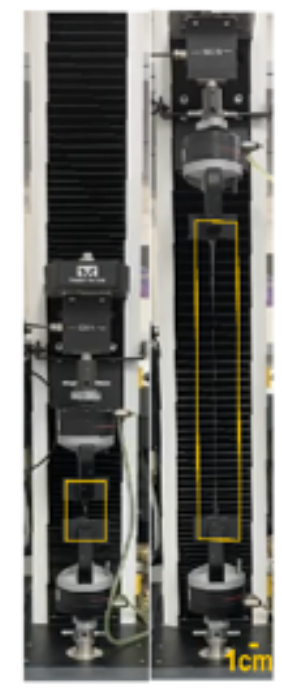

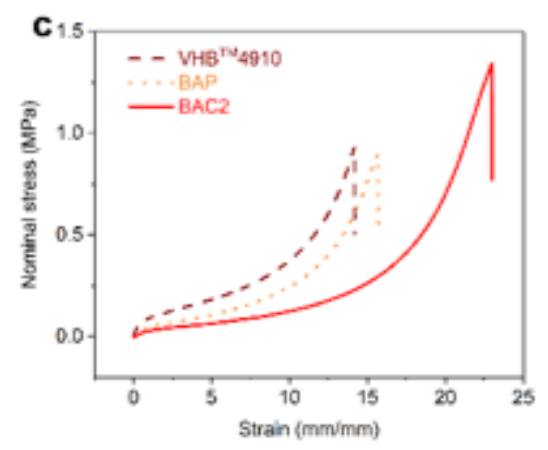
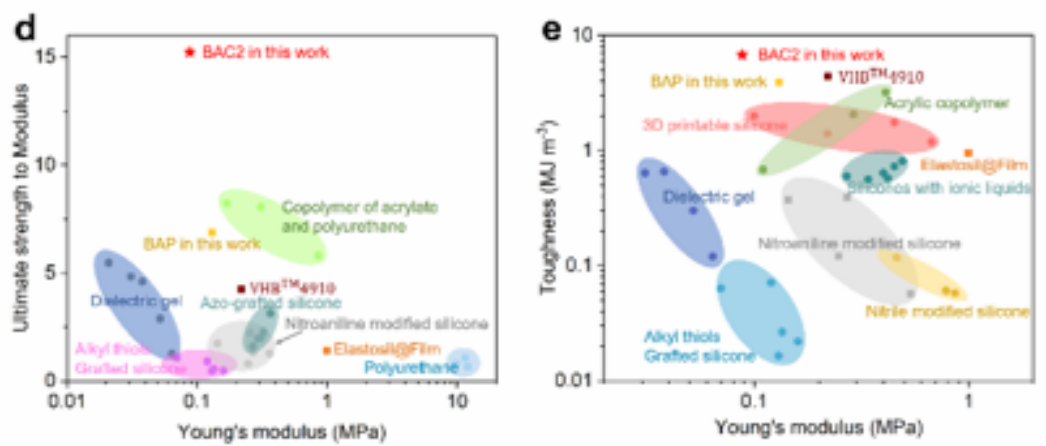

Figure 1

Performance of uniform hybrid polymer network. a, The precursor (ai, aiii) consists of n-butyl acrylate (BA) as monomers and micro-molecules (ai) or macro-molecules-CN9021NS- with two vinyl groups ($\mathrm{CH} \llbracket \mathrm{CH} 2$ ) (aiii) as crosslinkers. When exposed to ultraviolet light (UV-light), it is cured to a flexible network (aii, named BAP; aiv, named BAC2). Compared with micro-molecules, macro-molecular crosslinkers can greatly eliminate the effect of uneven dispersion of crosslinkers on polymer network structure, and tend to construct a more uniform one. b, Comparison of BAC2 before (left) and after (right) uniaxial stretching. C, The stress-strain curves of commercial VHBTM4910 and BAP, BAC2 at a stretch rate of $500 \mathrm{~mm}$ min-1. d, Comparison of ultimate strength to modulus among VHBTM 4910, BAP, BAC2, dielectric gel38, copolymer of acrylate and polyurethane39, azo-grafted silicone9, nitroaniline modified silicone27, polyurethane28, alkyl thiols grafted silicone25 and commercial Elastosil@Film25. e, Toughness plotted against Young's modulus for VHBTM 4910, BAP, BAC2, dielectric gel38, silicones with ionic liquids26, acrylic copolymer31, nitroaniline modified silicone27, nitrile modified silicone40, alkyl thiols grafted silicone25, 3D printable silicone41, and commercial Elastosil@Film25. 

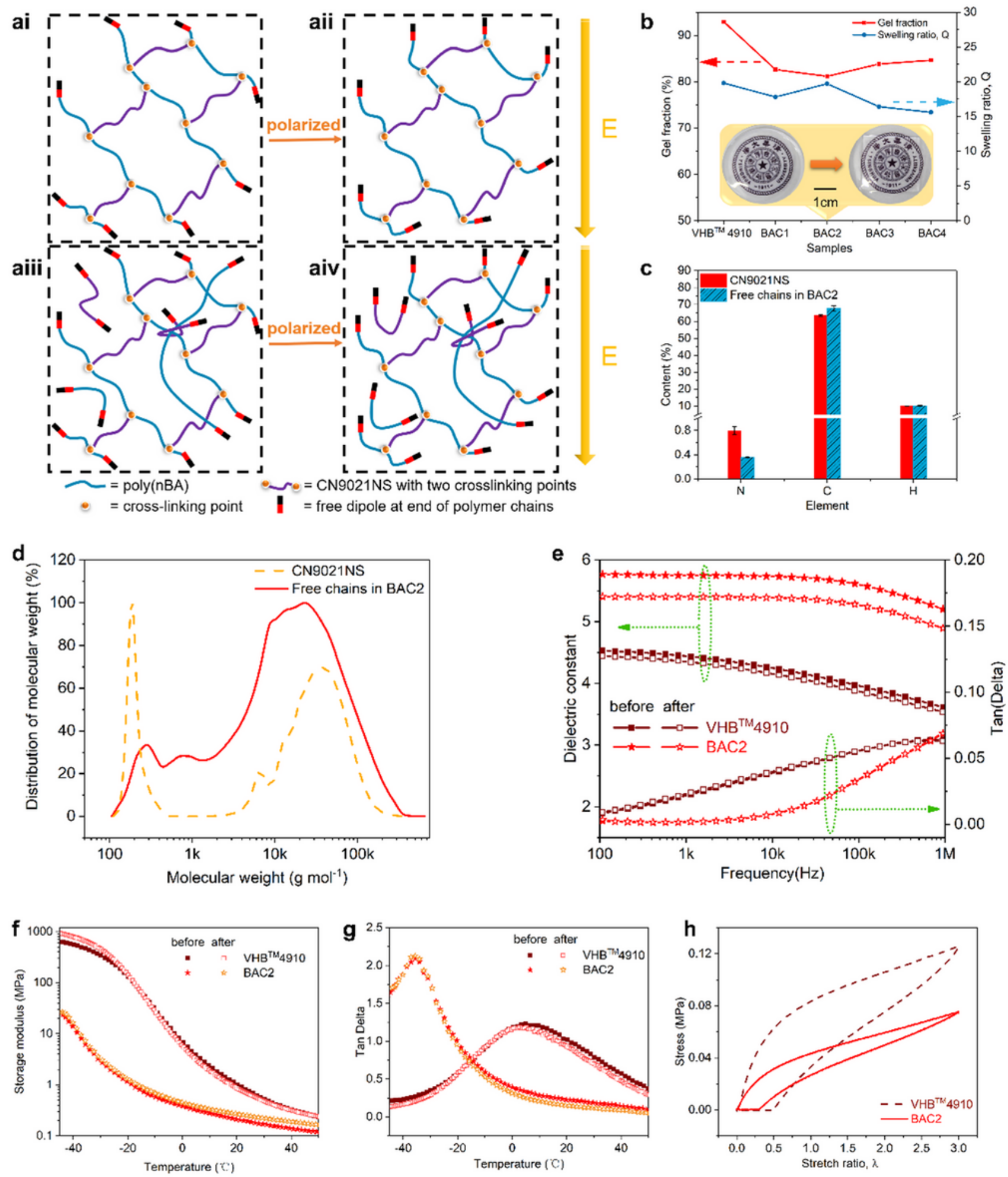

Figure 2

Influence of chemically uncrosslinked chains on dielectric and mechanical properties. a, Schematic comparison of the polarization process of acrylic elastomer without uncrosslinked chains (ai, aii) and BAC2 sample with a considerable amount of uncrosslinked chains (aiii, aiv). b, Gel fraction and swelling ratio of VHBTM4910 and synthesized elastomers. The insets show the optical images of BAC2 sample before and after swollen. c, Elemental analysis comparison between CN9021NS and free chains of BAC2 
after swollen. At least three samples were tested for the calculation of average value and standard deviation. d, Molecular weight distribution of CN9021NS and free chains of BAC2 after swollen. e, Frequency dependence of dielectric constant and dissipation factor (Tan $\mathrm{Q}$ ) of VHBTM4910 and BAC2 before and after swollen in the range of 102-106 Hz. f, g, Storage modulus (f) and mechanical loss (g) as a function of temperature of VHBTM4910 and BAC2 before and after swollen. $h$, The cyclic stress-strain curves of VHBTM4910 and BAC2 at a stretch rate of $500 \mathrm{~mm}$ min-1. The area encircled by the curve indicates the dissipated mechanical energy.

a

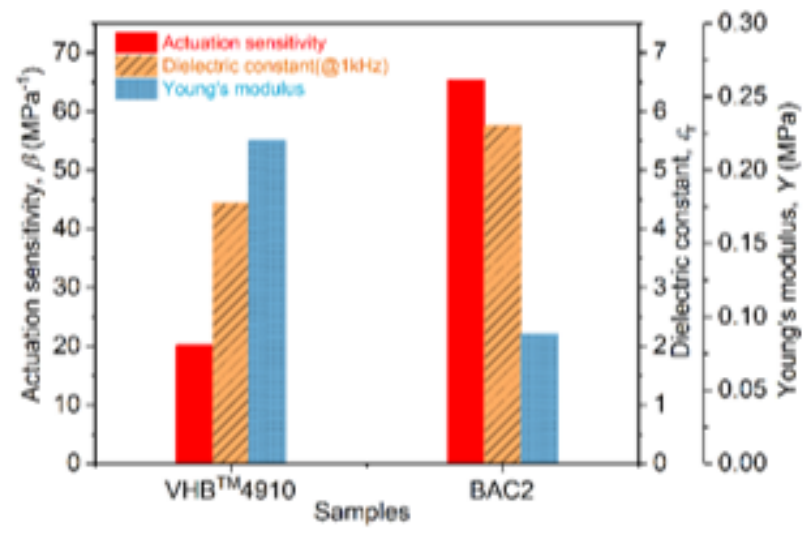

c

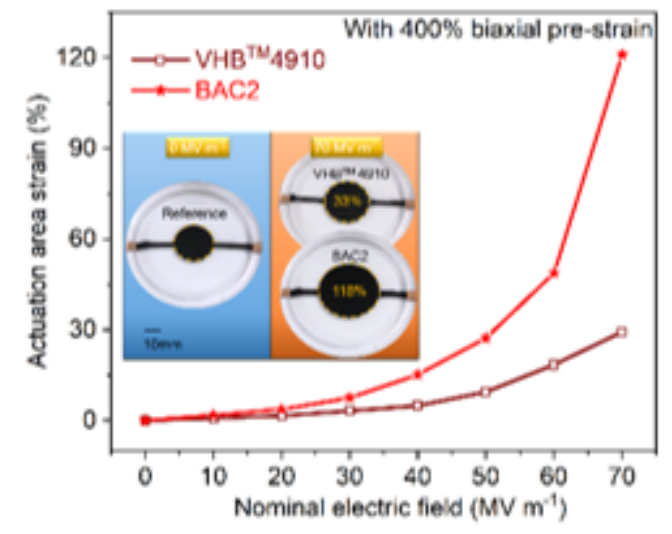

b

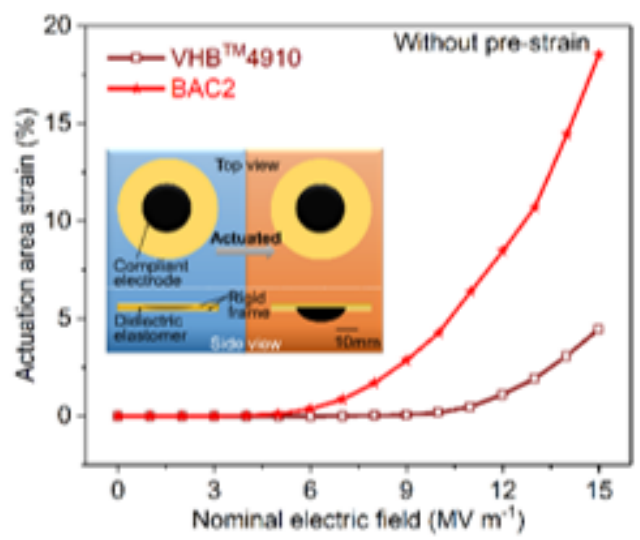

d

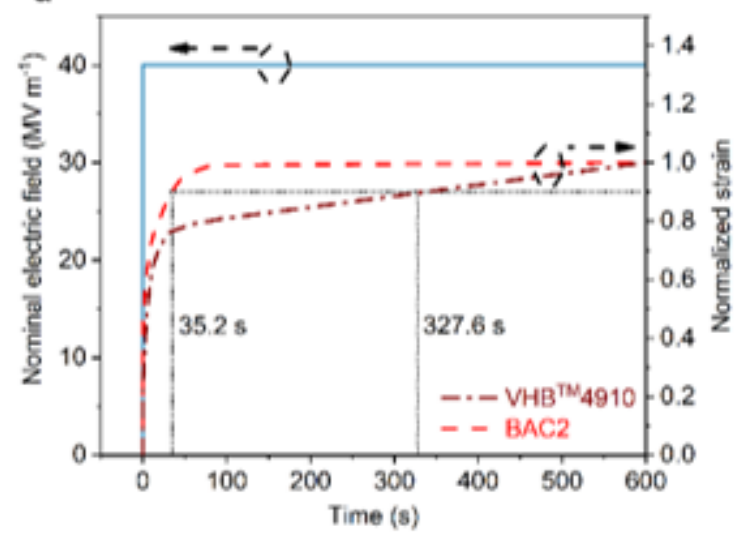

Figure 3

Actuation performance. a, Comparison of dielectric constant $\left(\varepsilon_{-} r\right)$ measured at $1 \mathrm{kHz}$, Young's modulus $(Y)$, and actuation sensitivity $(\square)$ between VHBTM4910 and BAC2 sample. b, Dependence of actuation area strain on the nominal electric field for the films of VHBTM4910 and BAC2 elastomers without prestrain. The nominal electric field is defined as voltage on films divided by initial thickness before electrically actuated. The inset shows the schematic diagrams illustrating the electro-actuation deformation process from the view of top and side directions. $c$, Dependence of actuation area strain on the nominal electric field for the films of VHBTM 4910 and BAC2 elastomers with $400 \%$ biaxial pre-strain. The inset displays the optical images of VHBTM 4910 and BAC2 films before and after actuated. $d$, Time dependent behavior of the actuated area strain of VHBTM 4910 and BAC2 films with $400 \%$ biaxial prestrain at $40 \mathrm{MV}$ m-1. Normalized strain denotes the time-varying strain divided by strain at $600 \mathrm{~s}$. 
a
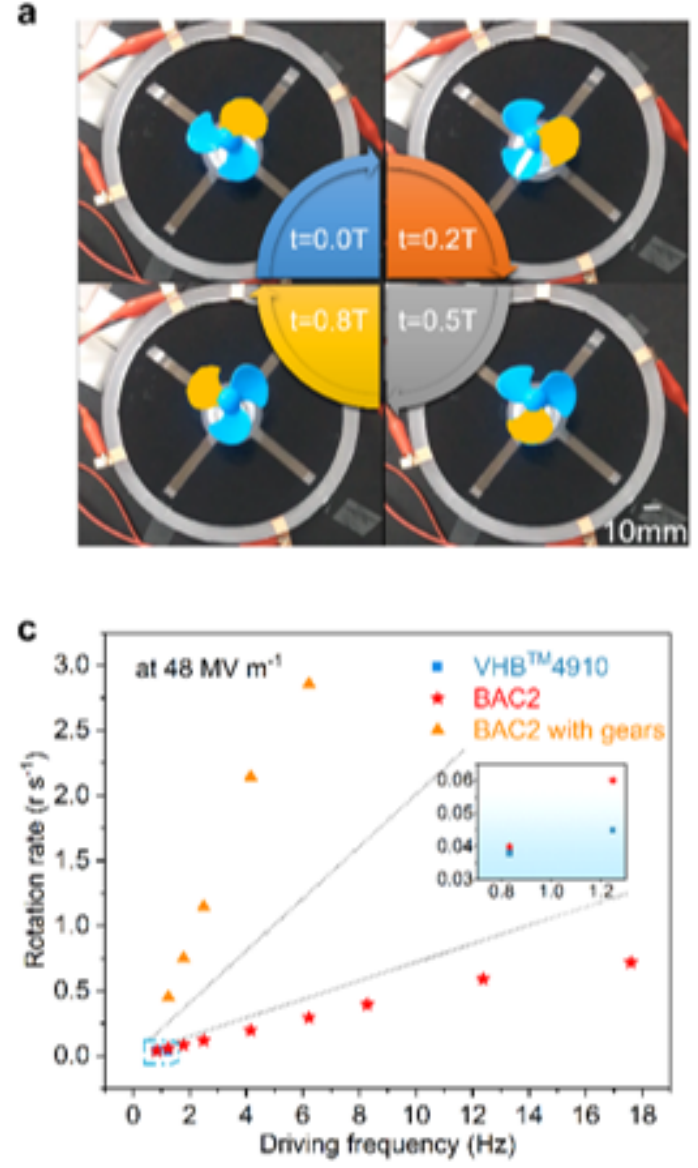

b

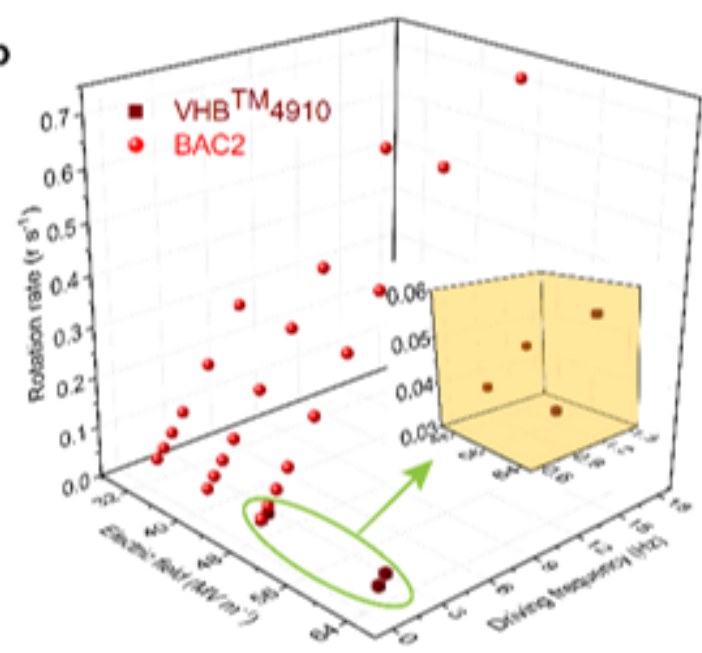

d

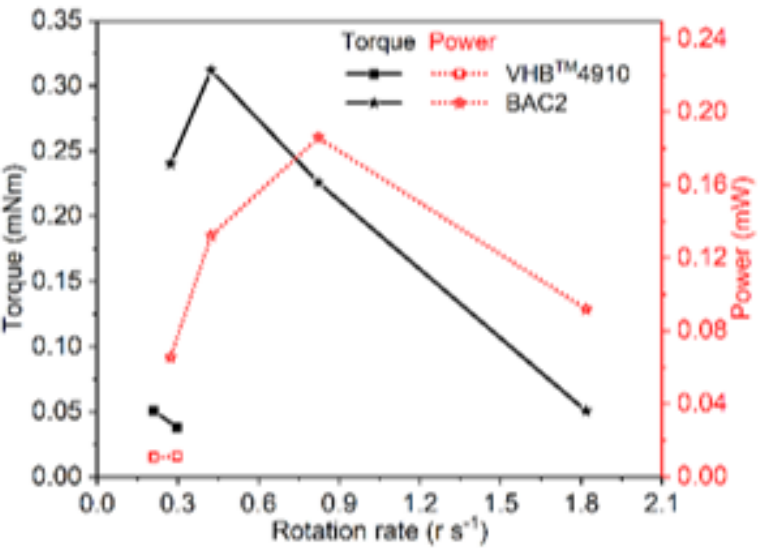

Figure 4

Mechanical output performance of soft non-magnetic motors made of dielectric elastomer films. a, Snapshots from the Supplementary Video 1 of a spinning non-magnetic motor displaying a complete rotation cycle, where $t$ and $T$ denote time and rotation period, respectively. $b$, Dependence of the rotation rate on electric field and driving frequency for the motors made of VHBTM 4910 and BAC2 films. The inset is a magnification of the data indicated by the arrow, which represents the electric field and frequency dependent behavior of the rotation rate for VHBTM4910 based motor. c, Dependence of the rotation rate on the driving frequency for the VHBTM 4910 and BAC2 based motors as well as BAC2

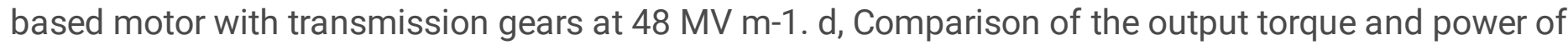
VHBTM 4910 and BAC2 based motors.

\section{Supplementary Files}

This is a list of supplementary files associated with this preprint. Click to download.

- SupplementaryVideo1.mp4

- SupplementaryVideo2.mp4

- SupplementaryVideo3.mp4 
- SupplementaryVideo4.mp4

- lijuanNCextendeddata.docx 